\title{
Negative Regulation of the Tight Junction Protein Tricellulin by Snail-Induced Epithelial-Mesenchymal Transition in Gastric Carcinoma Cells
}

\author{
Risayo Masuda ${ }^{a}$ Shuho Semba ${ }^{a}$ Eri Mizuuchi ${ }^{a}$ Kazuyoshi Yanagihara ${ }^{b}$ \\ Hiroshi Yokozaki ${ }^{a}$

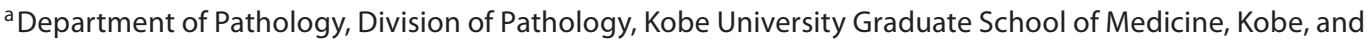 \\ ${ }^{b}$ Laboratory of Health Sciences, Department of Life Sciences, Faculty of Pharmacy, Yasuda Women's University, \\ Hiroshima, Japan
}

\section{Key Words}

Tricellulin · Snail • Epithelial-mesenchymal transition •

Gastric carcinoma

\begin{abstract}
Objective: Tricellulin plays a central role in the sealing of epithelia at tricellular contacts. We examined the effects of Snail, an epithelial-mesenchymal transition (EMT)-related transcription factor, on the regulation of tricellulin expression in human gastric carcinoma (GC)-derived cells. Method: Six human GC-derived cell lines were used in this study. Expression and localization of tricellulin was analyzed by reverse transcription (RT)-PCR and immunohistochemistry. Also, a Snail expression vector was transfected into HSC-45 cells to examine altered mRNA levels of tricellulin, E-cadherin, vimentin, $\mathrm{N}$-cadherin and several EMT transcription factors by quantitative real-time RT-PCR. Results: Abundant tricelIulin expression was detected in all GC-derived cells examined. In HSC-45 cells, transduction of Snail decreased the expression levels of tricellulin and E-cadherin but increased vimentin and $\mathrm{N}$-cadherin, which was accompanied by induction of EMT transcription factors such as Twist1, Twist2 and Slug.
\end{abstract}

In normal gastric mucosa, tricellulin protein was localized at the tricellular tight junction; however, in HSC-45 cells, tricellulin protein was distributed in the cytoplasm. In GC tissues, tricellulin expression at the cellular membrane was retained in a subset of EMT-negative GCs, and it disappeared in EMTpositive GCs. Conclusions: The findings in the present study suggest that repression of tricellulin expression may be related to Snail-induced EMT in human GCs.

Copyright $\odot 2010$ S. Karger AG, Basel

\section{Introduction}

The integrity of the epithelial cell layer that protects multicellular organisms from the external environment is maintained by intercellular junctional complexes composed of tight junctions (TJs), adherens junctions and desmosomes [1]. Among these junctional complexes, TJs act to prevent solute leakage through the paracellular pathway of epithelial cells [1]. To be more exact, TJs can be divided into 2 groups, i.e. bicellular TJs (bTJs), which are formed between 2 adjacent cells, and tricellular TJs (tTJs), which are formed where 3 cells meet. Occludin and

\section{KARGER}

Fax +4161306 1234 E-Mail karger@karger.ch www.karger.com
(C) 2010 S. Karger AG, Basel

$1015-2008 / 10 / 0772-0106 \$ 26.00 / 0$

Accessible online at:

www.karger.com/pat
Hiroshi Yokozaki, MD, PhD

Division of Pathology, Department of Pathology

Kobe University Graduate School of Medicine

7-5-1 Kusunoki-cho, Chuo-ku, Kobe 650-0017 (Japan)

Tel. +81 78382 5460, Fax +81 78382 5479, E-Mail hyoko@ @ed.kobe-u.ac.jp 
claudins have been identified as constituents of bTJ strands at cell-cell contact regions [2-4], and tricellulin was identified as being uniquely concentrated at tricellular contacts [5], in line with the fact that TJs completely disappear during the epithelial-mesenchymal transition (EMT) induced by Snail, a zinc finger type transcription factor [6, 7]. Human tricellulin consists of 546 amino acid polypeptides with 4 predicted transmembrane domains, and knockdown of tricellulin expression in epithelial cells was shown to diminish the tTJ network, consequently causing decreased transepithelial electric resistance and a size-selective disruption of the paracellular barrier [5]. These findings demonstrate that tricellulin is a key mediator for this epithelial barrier mechanism at the tricellular contacts.

Evidence has accumulated indicating abnormal expression of TJs in various human malignancies. A loss of claudin expression at the invasive front of advanced gastric carcinomas (GCs) results in higher malignancy grades with regard to potential metastatic ability and patient outcomes [8]. Similarly, a loss of claudin- 4 and claudin-7 is closely associated with the progression and development of esophageal and colorectal carcinomas [911]. In esophageal carcinoma, we have demonstrated Snail-induced EMT, which is accompanied by a decrease in epithelial markers (E-cadherin, claudin-1 and claudin7) and an increase in mesenchymal markers (vimentin) in vitro and in vivo [12]. Thus, the significance of bTJ-related proteins, including claudins, has been investigated to elucidate their association with carcinoma aggressiveness; however, the role of the $\mathrm{tTJ}$ protein tricellulin during the progression and development of GC remains unknown. In the present study, we investigated the effect of Snail-induced EMT on the regulation of tricellulin expression in GC-derived cells. In addition, expression of tricellulin in GC tissues was also examined to evaluate the association with EMT.

\section{Materials and Methods}

\section{Cell Lines and Gene Transfection}

Six GC-derived cell lines were used. HSC-45, HSC-57 and HSC-59 were established by one of the authors [12]. MKN-7 and MKN-74 were provided by Dr. Suzuki (Fukushima Medical University, Fukushima, Japan) [13]. TMK-1 was a gift from Dr. Yasui (Hiroshima University, Hiroshima, Japan) [14]. These cell lines were categorized into 3 types: well-differentiated GC, HSC-57, MKN-7 and MKN-74; poorly differentiated GC, HSC-59 and TMK-1, and Signet ring cell GC, HSC-45. Cells were maintained in RPMI-1640 (Invitrogen, Carlsbad, Calif., USA) supplemented with $10 \%(\mathrm{v} / \mathrm{v})$ fetal bovine serum. HSC-45 cells were also used
Table 1. Sequences of primers used in this study

\begin{tabular}{ll}
\hline $\begin{array}{l}\text { Tricellulin } \\
\text { Forward }\end{array}$ & 5'-CAT GAG GCA GCT CGG AGA CA-3' \\
Reverse & 5'-GTG TCC ACT CAG TAG TTC AGG-3' \\
\hline $\begin{array}{l}\text { E-cadherin } \\
\text { Forward }\end{array}$ & 5'-TGC CCA GAA AAT GAA AAA GG-3' \\
Reverse & 5'-GTG TAT GTG GCA ATG CGT TC-3' $^{\prime}$ \\
\hline Vimentin & \\
Forward & 5'-GAG AAC TTT GCC GTT GAA GC-3' \\
Reverse & 5'-TCC AGC AGC TTC CTG TAG GT-3' \\
\hline Snail & \\
Forward & 5'-GCG AAT TCT AGC GAG TGG TTC TTC \\
Reverse & TGC GCT ACT GCT-3' \\
& GAA GA GCG GCC GCC AGG TAT GGA GAG \\
&
\end{tabular}

\begin{tabular}{ll}
\hline Twist 1 & \\
Forward & $5^{\prime}$-AGCTACGCCTTCTCGGTCT-3' \\
Reverse & $5^{\prime}$-CCTTCTCTGGAAACAATGACATC-3'
\end{tabular}

\begin{tabular}{ll}
\hline Twist2 & \\
Forward & $5^{\prime}$-GCAAGAAGTCGAGCGAAGAT-3' \\
Reverse & $5^{\prime}$-GCTCTGCAGCTCCTCGAA-3'
\end{tabular}

\begin{tabular}{ll}
\hline Slug & \\
Forward & $5^{\prime}$-ATGCCGCGCTCCTTCCT-3' \\
Reverse & $5^{\prime}$-TGTGTCCAGTTCGCT-3'
\end{tabular}

$\begin{array}{ll}P d x-1 & \\ \text { Forward } & 5^{\prime} \text {-AAGTCTACCAAAGCTCACGCG-3' } \\ \text { Reverse } & 5^{\prime} \text {-GTAGGCGCCGCCTGC-3' }\end{array}$

$C d x-2$

Forward 5'-GGAACCTGTGCGAGTGG-3'

Reverse 5'-TTCCTCCGGATGGTGATGA-3'

\section{$N$-cadherin}

Forward 5'-GGTGGAGGAGAAGACCAG-3'

Reverse 5'-GGCATCAGGCTCCACAGT-3'

$\begin{array}{ll}\text { GAPDH } & \\ \text { Forward } & 5^{\prime} \text {-ACC ACA GTC CAT GCC ATC AC-3' } \\ \text { Reverse } & 5^{\prime} \text {-TCC ACC ACC CTG TTG CTG TA-3' }\end{array}$

for gene transfection experiments. The full-length human Snail cDNA (SNAI1, GDB accession No. NM_005985) was cloned into the pCX4bsr vector to generate pCX4-Snail expression vector [15]. pCX4-Snail and pCX4bsr empty vector were transiently transfected into HSC-45 cells using Lipofectamine 2000 (Invitrogen) according to the manufacturer's instructions. For the extraction of total RNA, the cells were collected $48 \mathrm{~h}$ after gene transfection.

Reverse Transcription-PCR Analysis

Total RNAs from each GC cell line were isolated using an RNeasy Mini kit (Qiagen, Hilden, Germany), and reverse tran- 
scription (RT)-PCR was performed with a OneStep RT-PCR assay kit (Qiagen). The primer sets were designed as shown in table 1. Each $25-\mu$ l reaction mixture containing $10 \mathrm{ng}$ of total RNA, $1 \mathrm{mM}$ of the primer pair and 0.75 units of reverse transcriptase and Taq DNA polymerase was amplified for 30 cycles with the following regimen: $\mathrm{RT}$ at $50^{\circ} \mathrm{C}$ for $30 \mathrm{~min}$, denaturation at $94^{\circ} \mathrm{C}$ for $30 \mathrm{~s}$, annealing at $58^{\circ} \mathrm{C}$ for $30 \mathrm{~s}$ and extension at $72^{\circ} \mathrm{C}$ for $1 \mathrm{~min}$. The RT-PCR products underwent electrophoresis in $2 \%$ agarose gel.

\section{Quantitative Real-Time RT-PCR Analysis}

Quantitative real-time RT-PCR was performed using the ABI Prism 7700 Sequence Detection System (Applied Biosystems, Foster City, Calif., USA) and the QuantiTect SYBR Green RT-PCR kit (Qiagen). The primer sets were designed as shown in table 1. After an initial incubation at $50^{\circ} \mathrm{C}$ for $30 \mathrm{~min}$ and denaturation at $95^{\circ} \mathrm{C}$ for $15 \mathrm{~min}$, the following cycling conditions (40 cycles) were used: denaturation at $94^{\circ} \mathrm{C}$ for $15 \mathrm{~s}$, annealing at $55^{\circ} \mathrm{C}$ for $30 \mathrm{~s}$ and elongation at $72^{\circ} \mathrm{C}$ for $30 \mathrm{~s}$. All of the experiments were performed in triplicate. The $\mathrm{Ct}$ for each gene from each sample was standardized to the sample GAPDH value $(\Delta \mathrm{Ct}=$ gene $\mathrm{Ct}-$ $G A P D H \mathrm{Ct}$ ), and this value was then compared between pCX4Snail-transfected and pCX4bsr-transfected cells using the $\Delta \Delta \mathrm{Ct}$ method $[\Delta \Delta \mathrm{Ct}=(\mathrm{pCX} 4-$ Snail-transfected $\Delta \mathrm{Ct})-(\mathrm{pCX} 4 \mathrm{bsr}-$ transfected $\Delta \mathrm{Ct}$ )], which was then used to determine the fold change $\left(=2^{-\Delta \Delta \mathrm{Ct}}\right)$.

\section{Immunocytochemistry and Immunohistochemistry}

For immunofluorescence, cells were grown on glass coverslips and then fixed with precooled methanol $\left(-20^{\circ} \mathrm{C}\right)$ for $10 \mathrm{~min}$. After washing with phosphate-buffered saline, cells were stained with antitricellulin (Zymed, South San Francisco, Calif., USA) and antioccludin (Santa Cruz Biotechnology, Santa Cruz, Calif., USA). Staining patterns obtained with antibodies against tricellulin and occludin were visualized with Cy2-conjugated antibody against rabbit IgG (GE Healthcare, Piscataway, N.J., USA) and Cy3-conjugated antibody against rabbit IgG (GE Healthcare), respectively. The nuclei were stained with 4',6-diamidino-2-phenylindole. The cells were examined with a confocal microscope (TSC SPE, Leica, Wetzlar, Germany).

For immunohistochemistry, a modified version of the immunoglobulin enzyme bridge technique with an LSAB kit (Dako, Glostrup, Denmark) was used. Briefly, deparaffinized and rehydrated sections were autoclaved in a citrate buffer. After blocking of endogenous peroxidase and nonspecific reactions, primary antibodies against tricellulin, Snail (Abcam, Cambridge, UK), Ecadherin (Transduction, Lexington, Ky., USA) and vimentin (Thermo Electron, Pittsburgh, Pa., USA) were applied to the sections, which were subsequently incubated with biotinylated monkey antirabbit IgG. Streptavidin conjugated to horseradish peroxidase was used to immerse the sections with 3,3'-diaminobenzidine tetrahydrochloride. The sections were counterstained with hematoxylin. The results of immunohistochemistry were evaluated by two pathologists (R.M. and S.S.). The immunoreactivities of tricellulin, Snail, E-cadherin and vimentin were graded according to the number of stained cells and the staining intensity in individual cells as follows: negative, i.e. almost no positive cells or $<50 \%$ of tumor cells showed weak immunoreactivity, or positive, i.e. $>50 \%$ of tumor cells showed weak immunoreactivity or tumor cells showed intense immunoreactivity.

\section{Results}

\section{Downregulation of Tricellulin Expression by Snail in GC Cells}

We first investigated the expression of tricellulin in GC cell lines. Among several isoforms of the tricellulin gene transcripts, we investigated expression of the full-length (GDB accession No. NM_001038603) and the exon 3 deletion variant (NM_144724) forms (fig. 1a). All the GC cell lines examined (HSC-45, HSC-57, HSC-59, MKN-7, MKN-74 and TMK-1) showed abundant expression of the full-length form, but expression levels of the exon 3 deletion variant of tricellulin were relatively low in these GC cells (fig. 1b). In addition, expression of E-cadherin was detected in all GC cells, whereas vimentin levels were undetectable (fig. 1b). Although HSC-57, HSC-59 and TMK1 expressed low to moderate levels of Snail, the other cells did not express Snail (fig. 1b); therefore, we considered that HSC-45, MKN-7 and MKN-74 cells were of the true epithelial phenotype (Snail $/$ E-cadherin ${ }^{+} /$vimentin $^{-}$) [15].

A gene transduction experiment was conducted to assess the possible correlation between the $\mathrm{tTJ}$ protein tricellulin and Snail-induced EMT. Of the true epithelial phenotype GC cell lines, HSC-45 (Snail-/E-cadherin ${ }^{+}$) vimentin ${ }^{-}$) cells were used for this experiment (fig. 1b, c). Results of quantitative real-time RT-PCR are summarized in figure 1d. Transduction of Snail increased the EMT-related transcription factors Twist1 (2.1-fold) [16], Twist2 (8.2-fold) [17] and Slug (20.9-fold) [18] at the mRNA levels; in contrast, the differentiation transcription factors Pdx-1 [19] and Cdx-2 [20] were downregulated (-2.0- and -20.9-fold, respectively). Simultaneously, tricellulin expression was repressed by the induction of Snail (-1.3-fold), as was E-cadherin (-1.7-fold). In contrast, vimentin and $\mathrm{N}$-cadherin were upregulated (1.4and 3.8-fold, respectively).

\section{Association between Reduced Expression of Tricellulin and Snail-Induced EMT in GC Tissues}

Next, the expression and localization of tricellulin protein was examined. The specificity of an antibody against human tricellulin was evaluated by detecting 64$\mathrm{kDa}$ bands by Western blotting (fig. 2a). In normal gastric mucosa, tricellulin was expressed in epithelial cells and was particularly localized at $\mathrm{tTJ}$ of the cellular membrane, as well as bTJs (fig. 2b, c). A vertical section revealed that tricellulin aggregated at the most apical side of the epithelia (fig. 2b, inset). Although localization of tricellulin protein in intestinal metaplasia of the stomach 


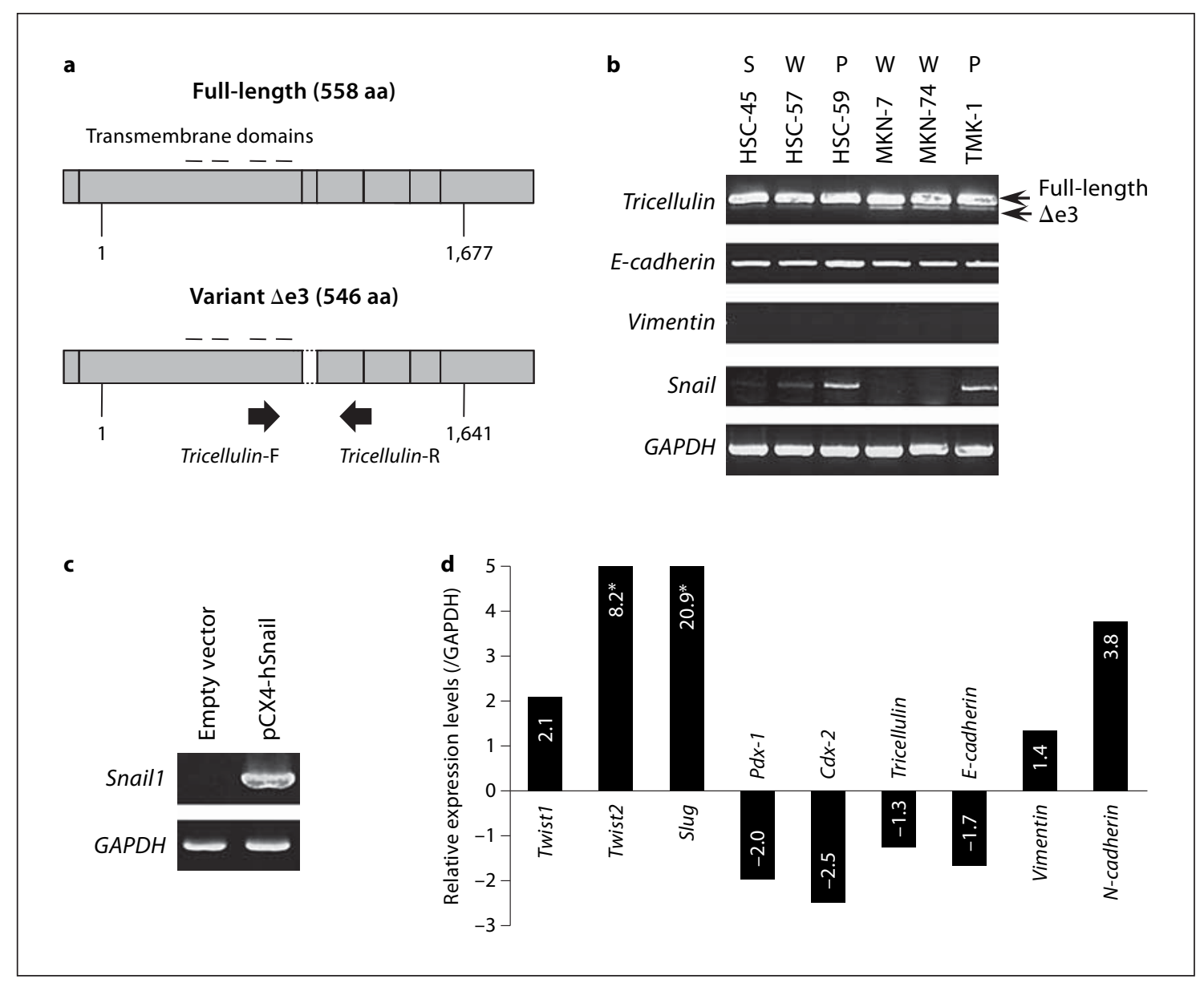

Fig. 1. a Schematic illustrations of full-length and exon 3-deleted $(\Delta \mathrm{e} 3)$ tricellulin gene transcripts. The arrows indicate the location of the primers used for RT-PCR and quantitative real-time RTPCR. The location of 4 predicted transmembrane domains is also shown. $\mathrm{F}=$ Forward; $\mathrm{R}=$ reverse. $\mathbf{b}$ Expression of tricellulin, $E$ cadherin, vimentin and Snail in GC cell lines. GAPDH expression levels were also examined as a control. GC-derived cell lines were categorized into 3 types: well-differentiated GC (W), HSC-57, MKN-7 and MKN-74; poorly differentiated GC (P), HSC-59 and TMK-1, and Signet ring cell GC (S), HSC-45. $\Delta \mathrm{e} 3=$ Exon 3 -de-

was also examined, there was no difference in the expression pattern and distribution of tricellulin in the presence or absence of intestinal metaplasia (data not shown). However, in HSC-45 cells, tricellulin expression was weakly repressed and was diffusely distributed in the cytoplasm but not at the cellular membrane (fig. 2e). We also detected a cytoplasmic distribution of tricellulin in the other GC cell lines (data not shown). Conversely, no significant differences were detected in the membrane expression of occludin, a bTJ-specific protein, in normal gastric mucosa and HSC-45 cells (fig. 2d, f). leted tricellulin variant. c Results of RT-PCR. Transduction of human Snail was confirmed. d Induction of ectopic Snail repressed tricellulin expression levels in HSC-45 cells. Relative expression of tricellulin, E-cadherin, vimentin and $N$-cadherin were determined according to the results of quantitative real-time RT-PCR. The mRNA expression levels of transcription factors (Twist1, Twist2, Slug, $P d x-1$ and $C d x-2)$ were also investigated. GAPDH levels were examined as a control. * The bars reach the highest limit.

Furthermore, we investigated tricellulin expression in GC tissue samples. Based on the results showing the altered levels and distribution of tricellulin in GC cells, we attempted to investigate the correlation of the tricellulin status with Snail-mediated EMT in GC tissues. According to the expression patterns of Snail, E-cadherin and vimentin, the status of EMT was determined in each GC case at the invasive front. EMT was strictly defined as occurring only when GC cells expressed Snail and vimentin but not E-cadherin (fig. 3a). Maintenance of tricellulin expression at the cellular membrane was detected in 

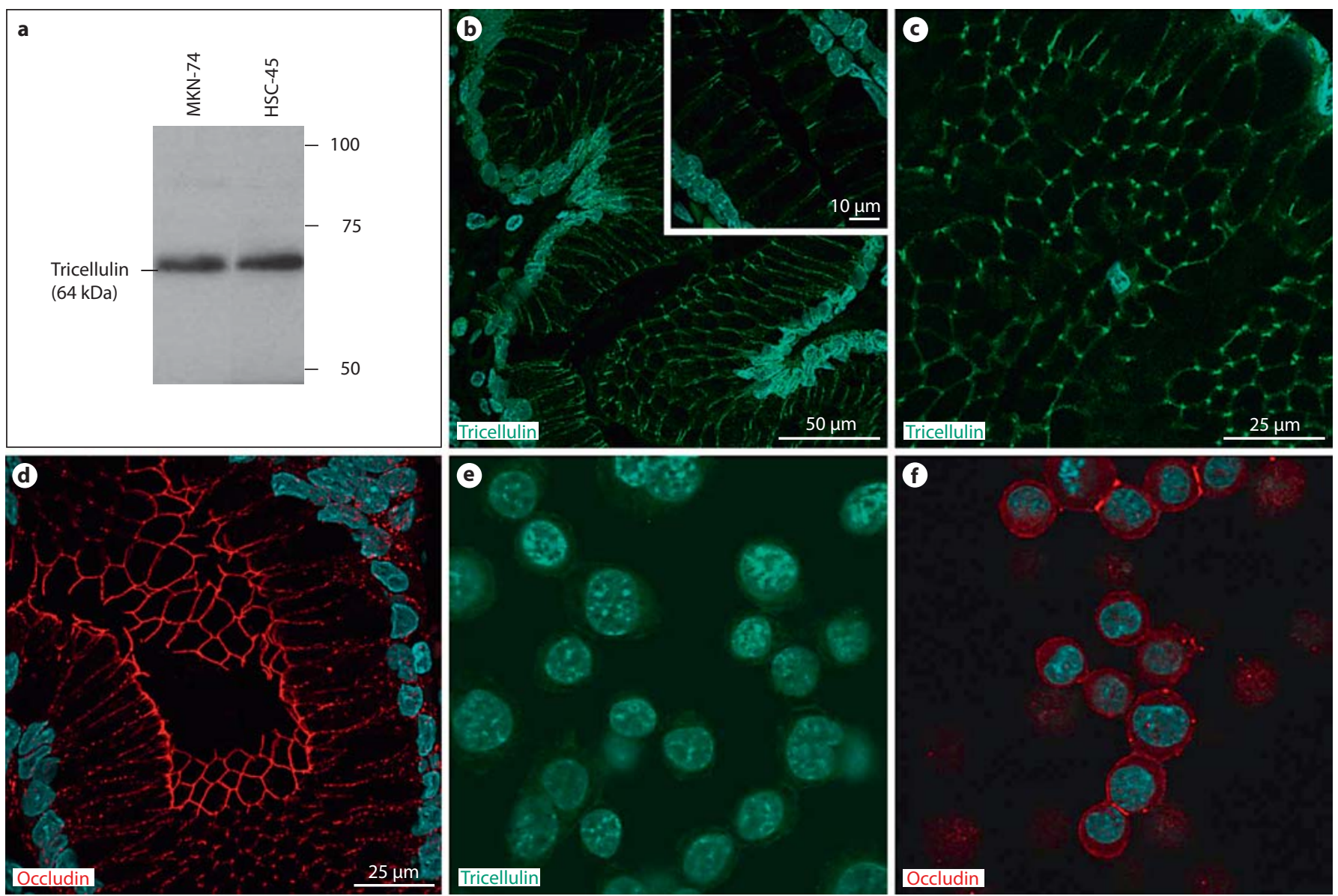

Fig. 2. a The specificity of an antibody against tricellulin was evaluated by Western blotting. b-d Results of immunofluorescence in nonneoplastic gastric epithelial cells. Localization of tricellulin (b, c; green) and occludin (d; red) was detected. Tricellulin was predominantly concentrated at the tTJs, particularly at the apical side of the epithelial cells (b, inset), while occludin was detected mainly at the bTJs. e, f Expression of tricellulin (green) and occludin (red) in MKN-74 cells was investigated. Tricellulin protein was diffusely distributed in the cytoplasm, whereas occludin was expressed at the cellular membrane of bTJs. Nuclei were stained with 4',6-diamidino-2-phenylindole (blue).
EMT-negative GC cases $\left(\right.$ Snail $^{+/-}$E-cadherin ${ }^{+} /$vimentin $^{+}$), which included well-differentiated GCs; however, in EMT-positive GC cases (Snail $/$ E-cadherin ${ }^{-} /$vimentin $^{+}$), in which poorly differentiated GCs were included, the membrane localization of tricellulin disappeared and weak immunoreactivity was detected in the cytoplasm (fig. 3b).

\section{Discussion}

It is widely accepted that the loss of cell-cell contact due to dysfunction or reduction of the adhesion molecules is one of a series of pivotal events in the process of carcinoma invasion and metastasis, allowing the liberation of individual carcinoma cells from the primary tumor. As with the adherence junction proteins, decreased levels of TJ proteins, particularly bTJ proteins such as claudins and occludin, are detected in various human malignancies, and this decrease consequently increases the grade of malignancy and the incidence of distant metastasis of GC cells [21,22]. To the best of our knowledge, this is the first report to examine tTJ-related protein expression in human carcinoma cells. Previous studies have demonstrated that a loss of bTJ protein expression correlates not only with diffuse-type GCs [23] but also with the dedifferentiation of GCs [8], both of which presumably increase the invasiveness and metastatic potential of 
a

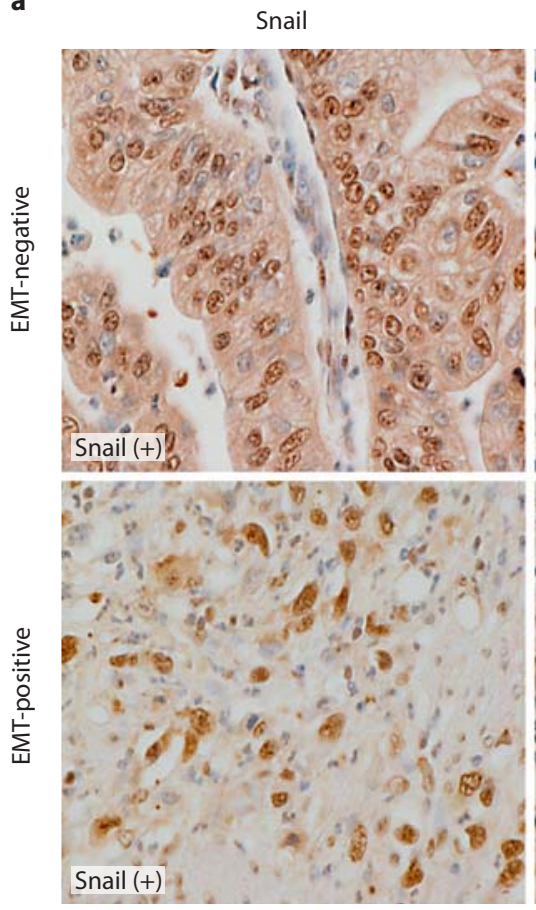

b

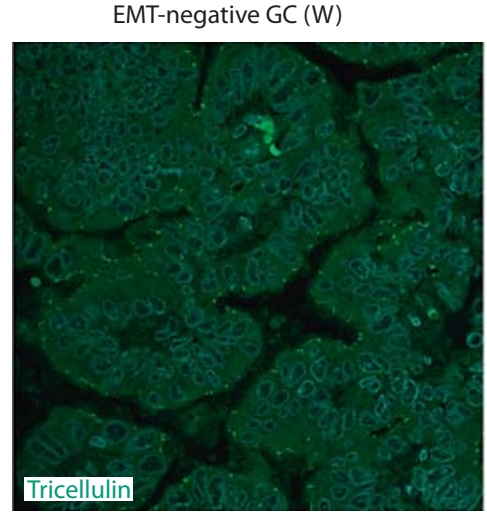

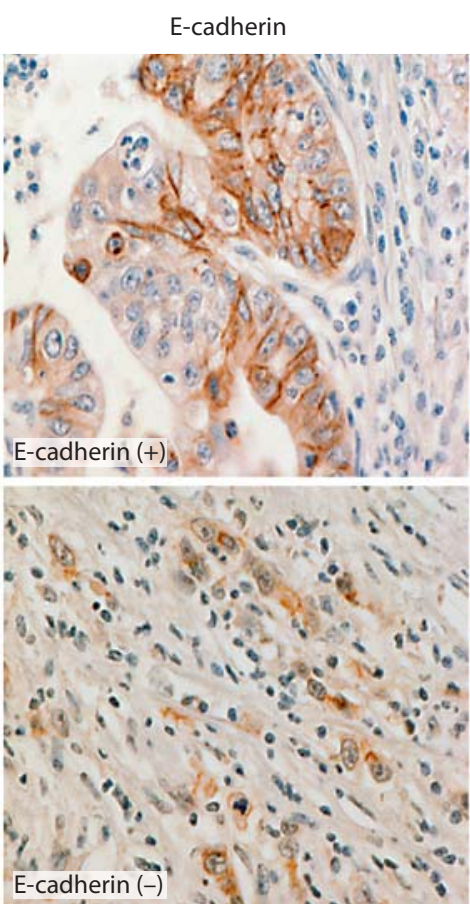
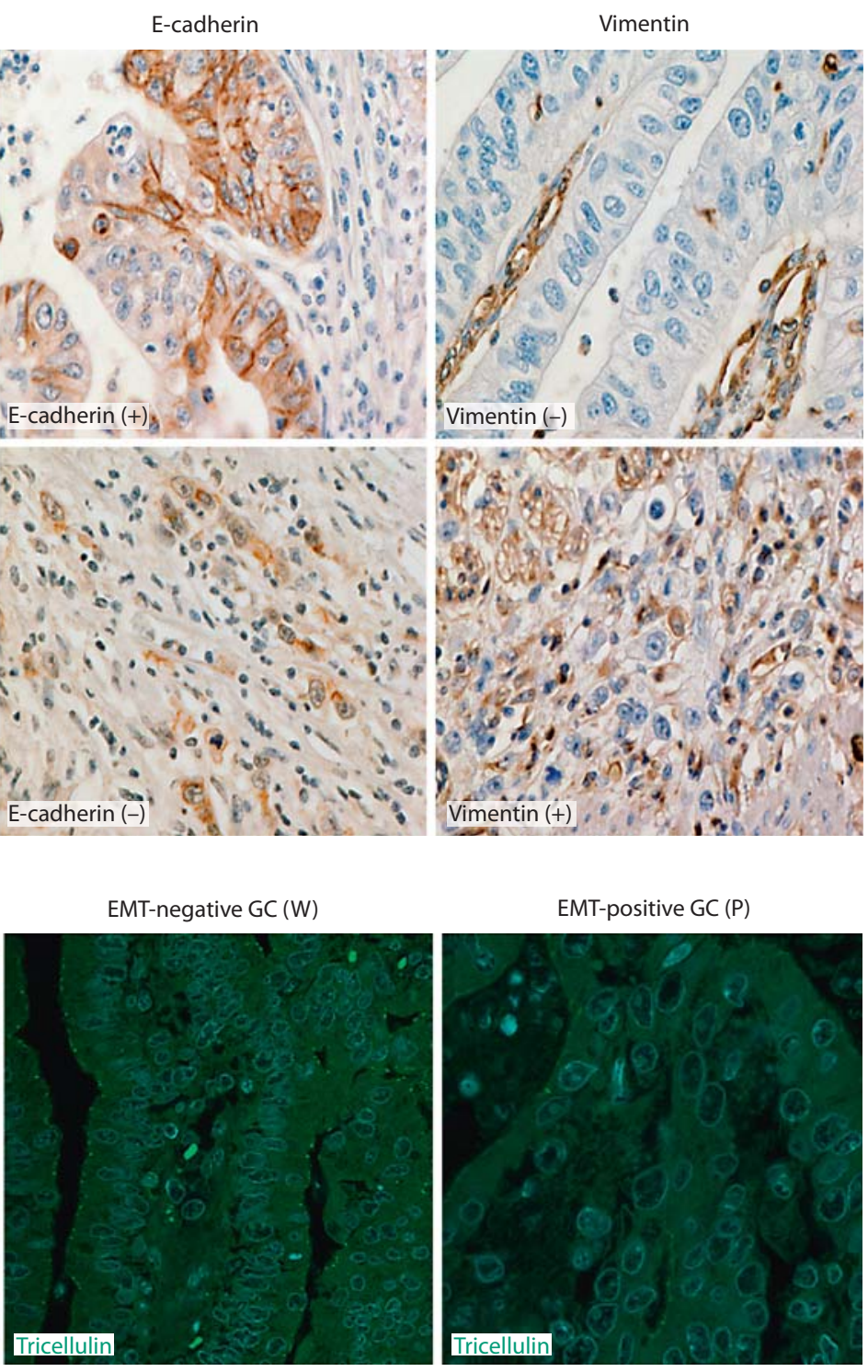

Fig. 3. a Evaluation of EMT at the invasive front of GCs. EMT was strictly defined as occurring only when tumor cells were E-cad-

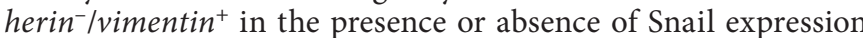
$\left(\right.$ Snail $^{+}$or $\left.^{-}\right)$. b Expression of tricellulin in human GC cases. Note that tricellulin expression was retained in tTJs in EMT-negative GCs; in contrast, membranous tricellulin protein expression disappeared in EMT-positive cases. $\mathrm{W}=$ Well-differentiated GC; $\mathrm{P}=$ poorly differentiated GC. the cancer cells. Also, as we have shown in the present study, decreased expression of tricellulin tends to be observed in poorly differentiated GCs, suggesting that the reduction of tricellulin and the resultant disruption of tricellular contacts may be associated with poorer differentiation and greater aggressiveness of GC cells. Recently, Ikenouchi et al. [24] demonstrated the physiological functions of tricellulin, as follows: (1) tricellulin is incorporated into claudin-based TJs independently of binding to zona occludens-1; (2) knockdown of occludin causes mislocalization of tricellulin at bTJs, and (3) tricellulin concentrates at tricellular contacts after the construction of bTJs. These data demonstrate the biological significance not only of tricellulin but also of the incorporation of TJ-related proteins into the process of bTJ and tTJ construction.

Snail is a zinc finger transcription factor that plays a central role in inducing the phenotypic transformation 
from epithelial cells to mesenchymal cells. In the process of EMT, Snail downregulates the expression of epithelial cell markers (e.g. E-cadherin, claudins and occludin) and upregulates mesenchymal markers (e.g. fibronectin and vimentin) [25]. In this study, we confirmed Snail-mediated suppression of tricellulin expression at the mRNA level in HSC-45 GC cells, which was accompanied by upregulation of vimentin expression. Interestingly, transduction of Snail upregulated other EMT regulators such as Twist1, Twist 2 and Slug (fig. 1d), in concordance with the report by Alves et al. [26] in which they demonstrated synergistic upregulation of Snail and Slug in GC cells. In GC tissue samples, as shown in figure 3a, Snail expression did not directly correlate with the loss of E-cadherin expression in the EMT-negative GCs, particularly in GC samples exhibiting a tubular structure. The induction of EMT is a sinister event during cancer progression and metastasis, acting as the first cascade allowing cells to delaminate from the primary tumor and to intravasate into lymphatic or venous vessels [27]. Snail induction and the resultant repression of E-cadherin expression are likely to be early events in tumor malignancy. Indeed, a close correlation between Snail expression and decreased E-cadherin expression has been documented in GCs [16], breast cancers [28] and colon cancers [29]. At the invasive front of GCs, we detected that the presence of EMT was closely correlated with downregulation and mislocalization of tricellulin immunohistochemically. As shown in figure $1 \mathrm{~b}$, the poorly differentiated GC cell lines HSC-59 and TMK-1 expressed relatively high levels of endogenous Snail. These findings suggest that induction of Snail at the invasive front may cause dysregulation of tricellulin and other cell-cell contact molecules, subsequently leading to the inhibition of gland formation of GC cells.

\section{Acknowledgments}

This study was supported by a Grant-in-Aid for Cancer Research (20-12) from the Ministry of Health, Labor and Welfare of Japan and Grants-in-Aid for Scientific Research (C-20590341 and C-19590347) from the Japan Society for Promotion of Science. We thank Prof. Mikio Furuse (Kobe University Graduate School of Medicine) for his advice.

\section{References}

1 Tsukita S, Furuse M, Itoh M: Multifunctional strands in tight junctions. Nat Rev Mol Cell Biol 2001;2:285-293.

\2 Furuse M, Hirase T, Itoh M, Nagafuchi A, Yonemura S, Tsukita S: Occludin: a novel integral membrane protein localizing at tight junctions. J Cell Biol 1993;123:1777-1788.

-3 Tsukita S, Furuse M: Occludin and claudins in tight junction strands: leading or supporting players? Trends Cell Biol 1999;9:268273.

-4 Furuse M, Sasaki H, Fujimoto K, Tsukita S: A single gene product, claudin-1 or -2, reconstitutes tight junction strands and recruits occludin in fibroblasts. J Cell Biol 1998;143: 391-401.

5 Hay ED: An overview of epithelio-mesenchymal transformation. Acta Anat (Basel) 1995;154:8-20.

6 Neito MA: The snail superfamily of zinc-finger transcription factors. Nat Rev Mol Cell Biol 2002;3:155-166.

-7 Ikenouchi J, Furuse M, Furuse K, Sasaki H, Tsukita S: Tricellulin constitutes a novel barrier at tricellular contacts of epithelial cells. J Cell Biol 2005;171:939-945.

8 Matsuda Y, Semba S, Ueda J, Fuku T, Hasuo T, Chiba H, Sawada N, Kuroda Y, Yokozaki $\mathrm{H}$ : Gastric and intestinal claudin expression at the invasive front of gastric carcinoma. Cancer Sci 2007;98:1014-1019.
-9 Usami Y, Chiba H, Nakayama F, Ueda J, Matsuda Y, Sawada N, Komori T, Ito A, Yokozaki $\mathrm{H}$ : Reduced expression of claudin-7 correlates with invasion and metastasis in squamous cell carcinoma of the esophagus. Hum Pathol 2006;37:569-577.

10 Ueda J, Semba S, Chiba H, Sawada N, Seo Y, Kasuga M, Yokozaki H: Heterogenous expression of claudin-4 in human colorectal cancer: decreased claudin- 4 expression at the invasive front correlates cancer invasion and metastasis. Pathobiology 2007;74:3241.

11 Nakayama F, Semba S, Usami Y, Chiba H, Sawada N, Yokozaski H: Hypermethylationmodulated down-regulation of claudin-7 expression promotes the progression of colorectal carcinoma. Pathobiology 2008; 75: 177-185.

12 Yanagihara K, Ito A, Toge T, Numoto M: Antiproliferative effects of isoflavones on human cancer cell lines established from the gastrointestinal tract. Cancer Res 1993;53: 5815-5821.

13 Hojo H: Establishment of cultured cell lines of human stomach cancer - origin and their morphological characteristics (in Japanese). Niigata Igakkai Zasshi 1977;91:737-752.
14 Ochiai A, Yasui W, Tahara E: Growth-promoting effect of gastrin on human gastric carcinoma cell line TMK-1. Jpn J Cancer Res 1985;76:1064-1071

15 Usami Y, Satake S, Nakayama F, Matsumoto M, Ohnuma K, Komori T, Semba S, Ito A, Yokozaki H: Snail-associated epithelialmesenchymal transition promotes oesophageal squamous cell carcinoma motility and progression. J Pathol 2008;215:330-339.

16 Rosivatz E, Becker I, Specht K, Fricke E, Luber B, Busch R, Höfler H, Becker KF: Differential expression of the epithelial-mesenchymal transition regulators snail, SIP1, and twist in gastric cancer. Am J Pathol 2002;161: 1881-1891.

-17 Ansieau S, Bastid J, Doreau A, Morel AP, Bouchet BP, Thomas C, Fauvet F, Puisieux I, Doglioni C, Piccinin S, Maestro R, Voeltzel T, Selmi A, Valsesia-Wittmann S, Caron de Fromentel C, Puisieux A: Induction of EMT by twist proteins as a collateral effect of tumor-promoting inactivation of premature senescence. Cancer Cell 2008;14:79-89.

18 Savagner P, Yamada KM, Thiery JP: The zinc-finger protein slug causes desmosome dissociation, an initial and necessary step for growth factor-induced epithelial-mesenchymal transition. J Cell Biol 1997;137:14031419. 
-19 Buettner M, Dimmler A, Magener A, Brabletz T, Stolte M, Kirchner T, Faller G: Gastric PDX-1 expression in pancreatic metaplasia and endocrine cell hyperplasia in atrophic corpus gastritis. Mod Pathol 2004;17:56-61.

-20 Silberg DG, Sullivan J, Kang E, Swain GP, Moffett J, Sund NJ, Sackett SD, Kaestner KH: $\mathrm{Cdx} 2$ ectopic expression induces gastric intestinal metaplasia in transgenic mice. Gastroenterology 2002;122:689-696.

-21 Kimura Y, Shiozaki H, Hirao M, Maeno Y, Doki Y, Inoue M, Monden T, Ando-Akatsuka Y, Furuse M, Tsukita S, Monden M: Expression of occludin, tight-junction-associated protein, in human digestive tract. Am J Pathol 1997;151:45-54.
22 Sanada Y, Oue N, Mitani Y, Yoshida S, Nakayama H, Yasui W: Down-regulation of the claudin-18 gene, identified through serial analysis of gene expression data analysis, in gastric cancer with an intestinal phenotype. J Pathol 2006;208:633-642.

23 Soini Y, Tommola S, Helin H, Martikainen P: Claudins 1, 3, 4 and 5 in gastric carcinoma loss of claudin expression associates with the diffuse type. Virchows Arch 2006;448:5258.

24 Ikenouchi J, Sasaki H, Tsukita S Furuse M, Tsukita S: Loss of occludin affects tricellular localization of tricellulin. Mol Biol Cell 2008; 19:4687-4693.

25 Barrallo-Gimeno A, Neito MA: The Snail genes as inducers of cell movement and survival: implications in development and cancer. Development 2005;132:3151-3161.

-26 Alves CC, Rosivatz E, Schott C, Hollweck R, Becker I, Sarbia M, Carneiro F, Becker K-F: Slug is overexpressed in gastric carcinomas and may act synergistically with SIP1 and Snail in the down-regulation of E-cadherin J Pathol 2007;211:507-515.
27 Thiery JP: Epithelial-mesenchymal transitions in tumour progression. Nat Rev Cancer 2002;2:442-454.

28 Blanco MJ, Moreno-Bueno G, Sarrio D, Locascio A, Cano A, Palacios J, Neito MA: Correlation of Snail expression with histological grade and lymph node status in breast carcinomas. Oncogene 2002;21:3241-3246.

-29 Pálmer HG, Larriba MJ, García JM, Ordóñez-Morán P, Peña C, Peiró S, Puig I, Rodríguez R, de la Fuente R, Bernad A, Pollán M, Bonilla F, Gamallo C, de Herreros AG, Muñoz A: The transcription factor SNAIL represses vitamin D receptor expression and responsiveness in human colon cancer. Nat Med 2004;10:917-919. 